\title{
Meeting Human Development Goals with Low Emissions
}

\author{
Jiahua Pan*
}

\section{Introduction}

Greenhouse gas (GHG) emissions arise from human economic activities and serve the ultimate purpose of human development. Some emissions are essential for basic needs while others, for extravagant or wasteful purposes, are unnecessary and even in conflict with human development.

Given the linkage of emissions to human development, mitigation options for developing countries will have to look first at their impact on development and then at their effectiveness of emissions reductions. A variety of options (see, e.g. Baumert 2002; Hoehne et al. 2003; Aldy et al. 2003) have been published in literature, targeting either emissions or policies and measures (PAMs). Emissions targets are specified in either absolute (Kyoto-type) carbon emission reduction or in relative (intensity) terms. Various policies and measures are proposed and their impacts on or implications for carbon emissions predicted and analysed.

This article suggests that a focus on emissions targets may simply ignore development goals. But existing development policies and measures do not necessarily imply low emissions paths. For developing countries, are there options that meet human development goals and at the same time aim at emissions reductions? This article suggests an approach that would allow human development goals to be met but with low emissions avoiding the simple emissions limits or reduction targets, reflected in the Kyoto Protocol.

\section{Reconsideration of the Kyoto- style emission targets: from Berlin to Marrakech}

The existing international emission mitigation frameworks are directly designed for stabilising the carbon concentration in atmosphere. But experiences since 1995 when the first UN Framework Convention on Climate Change
(UNFCCC) Conference of the Parties (COP 1) agreed that developed countries should have targets have been rather frustrating. To control climate change, it is necessary to limit GHG emissions, but GHG targets have often been sacrificed for other policy goals at higher priority level. It is imperative to reconsider the suitability of taking carbon targets as a goal for the global community.

When the UNFCCC came into legal effect in 1994, it was envisaged that deep cuts of GHG emissions would be realised. The political goodwill for ambitious targets was expressed in the negotiations conducted pursuant to the Berlin Mandate adopted at COP 1, which led to the Kyoto Protocol. In June 1997, shortly before the adoption of the Kyoto Protocol by COP 3 in 1997, the European Union (EU) leaders suggested, for example, a 15 per cent reduction relative to 1990 emissions levels.

However, the actual target for the richer countries (called Annex I Parties) in Kyoto Protocol, is 5.2 per cent reduction in aggregate by Annex I Parties based on their 1990 levels, ranging from 10 per cent increase to 8 per cent decrease for different members (the EU has an 8 per cent reduction target). After the Kyoto target was agreed in 1997 , three important Annex I Parties declined to implement their Kyoto commitments: the USA refused to join the Protocol in early 2001, followed by Australia. Although almost all the other Annex I Parties have accepted Kyoto by ratifying it, along with most of the developing countries (current ratifications stand at 120 countries covering threequarters of the world's population) Russia has not ratified the Protocol which means it has not yet entered into force.

It is clear that for these non-Kyoto countries (and many others whose emissions are rising notwithstanding their ratification of the Kyoto Protocol) that GHG mitigation is not the top priority target in their decision making. In fact, for both 
Figure 1: An overall framework for commitment

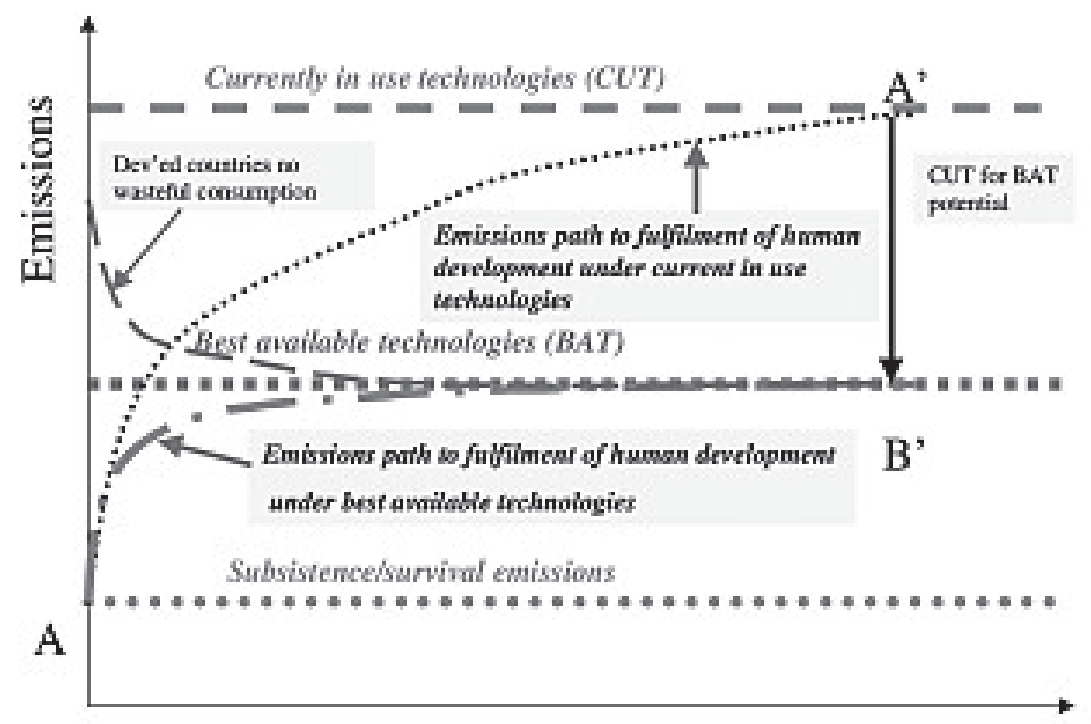

Time

developed and developing countries, there are many other even higher priority goals such as those related to political and/or social stability, economic growth or development as well as environmental pollution control/natural conservation. Climate change may be considered a subset of these goals and should be subject to the requirement of higher order goals. Because lower-level goals are easily disregarded when they conflict with higher-priority goals, it would, in fact, be more beneficial for GHG mitigation options to be linked to higher-priority human development rather than lower-level carbon constraints which tend to get ignored in political decision making.

\section{A human development approach to emissions reductions}

This section outlines a human development approach to setting international commitment for emissions reductions. Carbon emissions are remarkably different from other conventional pollutants because of their multiple nature. At least three different ways of seeing carbon emissions are possible. First, carbon emission can be considered both as a commodity and as a right. When they are linked to the achievement of basic human needs, carbon emissions fall into the category of human rights, which it is argued should not be transferable (see Pan 2003). However, if they are regarded as a commodity, they can be regarded as tradeable. Second, carbon emissions can be used for both collective goals and household consumption. Third, carbon emission is both a public good and a public ill. As a public good, it generates utility to individuals and the society. As a public ill, it produces such negative externalities as global warming.

This article suggests that the Kyoto Protocol will fail to function effectively because of its intrinsic weakness in not recognising these different types of carbon emissions: carbon should not be made a target in its own right, as Kyoto does, but rather should only be a target that is consistent with other development goals. Under the human development three types of emissions can be identified: (1) emissions for basic needs satisfaction; (2) collective consumption, and (3) luxurious/wasteful emissions. All these emissions are relevant to human development and they are discussed further below.

\subsection{Consumption of carbon emissions}

The goods and services consumed by households can be classified into two groups: basic needs and 
luxuries. A decent living standard would require the consumption of necessary calories for survival, shelter, basic healthcare and education, and access to clean water and commercial energy. Luxurious consumption includes living space larger than necessary, large cars when smaller ones can accommodate travel purposes and excessive heating and cooling etc.

Infrastructure is a major category of public goods, such as roads, railways, undergrounds, public utilities, airport, water supply and treatment facilities, and flood control and drainage systems. All these need such energy intensive materials as steel, cement and chemicals in addition to heavy machineries for their construction. Other examples include hospitals, schools and public office buildings.

Among the carbon demands associated with these final consumptions, the carbon for basic needs (Cbasic) is fixed $C^{\text {basic }}$, but that for luxurious consumption (Clux) is unlimited; carbon for infrastructure (Cinfrast) can be substantial but once it is constructed ( $\left.C^{\text {infrast }}\right)$, its maintenance does not require much additional carbon. Therefore, the total emission in terms of final consumption ( $C_{\text {total }}$ is:

$C_{\text {total }}=C_{\text {infrast }}+\mathrm{C}_{\text {basic }}+\mathrm{Clux}_{\text {lux }}$

As $C_{\text {infrast }} \leq C^{\text {infrast. }}$ Cbasic $\leq C^{\text {basic; }}$; $C_{\text {lux }} \rightarrow \infty, C^{\text {total }} \leq C^{\text {infrast }}$ $+C^{\text {basic }}$ if Clux is excluded.

This way of conceiving carbon constraints suggests that a country should be able to make a commitment limiting its emission at $C^{\text {total }}$, where this is specifically designated to $C_{\text {infrast }}$ and $C_{\text {basic }}$ for human development without jeopardising human development The total constraints set by this level of emissions can then be traced back to contributing industrial sectors.

$C^{\text {total }}$ can be treated as a right to a decent living standard but no ethical ground seems to justify Clux emissions. Therefore, luxury emissions should be discouraged. Cinfrast is for collective consumption of current and future generations as highways, railways, airports and many buildings can be used for centuries. Cbasic is necessary for human survival and a decent life. It is assumed that achievement of human development potential by the current generation is beneficial to future generations.

\subsection{Aligning human development with environmental constraints}

The above emission categorisation can function as a basis for allocating emission credits and for trading.
Emissions demand for basic needs could exceed the GHG emission limit to guarantee atmospheric concentration stabilisation. Because no compromise on the fulfilment of human development goals is allowed, it is necessary to seek low emission paths in the course of development goal realisation. Figure 1 conceptualises such a possibility.

Suppose that the human development goal is fixed at a decent living standard without any luxurious/wasteful emissions. As this goal is a priority and must be achieved, the constraint by carbon emissions is not considered. Low/zero carbon energy possibilities are technically viable in developed countries. Developing countries usually utilise traditional carbon intensive technologies because of capital and technology shortages (CUT curve as shown in Figure 1) and their emissions level can take a trajectory AA'. However, this trajectory can be lowered to $A B^{\prime}$ if technologies of higher energy efficiency (BAT as shown in Figure 1) are made available. Therefore, low carbon emissions can be achieved without lowering human development goals. Low carbon approaches include: (1) economic restructuring that is less carbon intensive; (2) energy mix restructuring to move to zero carbon-emitting energy forms; (3) improvement of energy efficiency; (4) use of carbon sinks; and (5) social policies such as family planning and poverty elimination.

In Figure 1, excessive emissions are discouraged and emissions from the developed economies will fall. For many least developed countries (LDCs), their current emission level is at subsistence level, much lower than a decent living standard.

\section{Defining commitments to implement the human development approach}

How can countries commit to implementing the many options that exist for low emissions paths? This article suggests that some of the options can be undertaken independently where others may require certain conditions being met. In general, three types of emissions reductions efforts may be identified: voluntary, conditional and obligatory. It is possible to transform the voluntary part into obligatory if emissions reductions can be made with certainty. Conditional reductions can be granted only to developing countries (because developed countries have their own resources to achieve reductions). Obligatory reductions restricting 
excessive/wasteful emissions of greenhouse gases can accrue to all countries whether developed or developing.

\subsection{Voluntary reductions}

Two factors contribute to automatic emission reduction without any climate policy intervention: technological progress and institutional innovation. For instance, energy demand elasticities in the industrialisation process of developed nations were one ( 1 per cent increase in GDP would require 1 per cent increase in energy consumption) or higher. But in the case of China, the figure has been only $\sim 0.5$ (1 per cent increase in GDP is realised with 0.5 per cent increase in energy consumption) during the last three decades. Institutional factors also play an important role in promoting emission reductions. With increasing awareness of climate changes, consumers may voluntarily adjust their behaviour towards living styles with lower energy and carbon consumption. Institutional arrangements such as emission standards and policy initiatives can also be made to stimulate low emissions.

As both trends can be considered likely to continue without much further climate-specific policy interventions, a country can make voluntary reduction commitments in accordance with the rate of automatic energy efficiency improvement. The reductions are voluntary in the sense they can be undertaken without further policy interventions, which would require specific obligations for particular actors, and also do not need external financial and technological support.

However, because developed economies play a leading role in the research and development (R\&D) of energy efficiency and low carbon energy source technologies, it may be possible to regard part of these resulting reductions as "obligatory" (in the sense that for these countries, such emissions reductions can be achieved with some certainty).

\subsection{Conditional reductions}

The term "conditional" in this context means reductions occur subject to the following three conditions: (1) reductions of emissions are based on the condition that developed country parties transfer of technologies or offer financial assistance to developing countries; (2) emissions reductions are conditional on not compromising human development goals but also do not encourage luxurious /wasteful emissions in the recipient country; and (3) to avoid creation of "hot air" (i.e. reduction credits that do not correspond to actions taken pursuant to climate or other reduction policies) credits for emissions reductions are conditional on actual progress being made to fulfil human development goals.

In essence, these conditions are rather similar to those in place for developing countries in the 1987 Montreal Protocol for the replacement of ozone depletion substances (ODS). Under the Montreal Protocol, developing countries' basic domestic need for ozone depleting substances is relevant to defining their reduction commitments. The phase-out of ODS in developing countries is also made conditional on technology transfer and financial assistance from the developed nations. With such assistance, China has now successfully phased out most of the production and consumption of chlorofluorocarbons (CFCs) and halons.

\subsection{Obligatory reductions}

For human development and global environmental sustainability, basic need satisfaction is part of human rights and should not be compromised. But at the same time, consumption resulting in excessive emissions must be restricted. Therefore, the obligation here has to be made in two aspects: (1) human basic needs satisfaction and (2) excessive/wasteful emission restriction. No distinction should be made between developed or developing country in this regard. For all human beings and communities, emissions for basic needs must be protected and excessive/wasteful emissions must be discouraged.

To make the approach operational, a practical problem is the precise definition of "luxurious/wasteful emissions". Despite the fact that circumstances differ greatly among nations and cultures, it is suggested here that there should be no discrimination against any particular nation or culture. A simple criterion such as world average or 120 per cent times world average might be used for some initial exercises. In other cases, such as nutritional requirements, scientific figures can be employed. The essential point is to discuss emissions as ones which serve basic needs and ones which can be considered beyond these. Most countries now have some kind of welfare system or other provisions which set a basic standard of living. Working out a basic level of emissions is not that far removed. 


\section{Environmental effectiveness of the human development approach}

The goal of human development must be consistent with stabilisation of GHG concentrations in the atmosphere (as otherwise human development is itself compromised). How can we fulfil human development potentials without surpassing carbon concentration limits? Answering this question means quantifying the amount of emissions resulting from specific pathways and options. Incentives for effective emissions reductions can then be devised and implemented.

Human development goals have to be linked to emissions reductions by specific actors. One practical way is to assess and translate national development goals into energy demand and emissions requirements. Some of the development process is highly predictable. Pan (2002) has looked at the rural energy transition process from traditional bio-fuels to fossil fuel dominated commercial energy in China. In making energy demand projections, Zhou et al. (2003) have developed three different scenarios for understanding energy demand in 2020. In all the scenarios, automatic energy efficiency improvement and new technologies are included in the calculation of energy demand and emissions. These can be the basis for desk analysis on the effectiveness of emissions reductions using the normative framework suggested above. This article does not provide empirical illustrations of this approach (because the technical details required are yet readily available). In general, the following steps could be followed.

\section{Step 1: Assessment of development goals \\ Many countries make comprehensive medium- and long-term development plans. The task here is to assess (1) whether the development goals of a country are consistent with human needs; (2) if there are wasteful/luxurious development projects; and (3) how the goals are linked to reinforce human development. The commitment period, over which the reductions are to be achieved, can be made consistent with duration of development planning, e.g. five years.}

\section{Step 2: Specification of socio-economic and environmental targets}

After assessment of development goals, socioeconomic and environmental targets can be identified, which would include economic growth rate, population features, welfare improvement, environmental protection, etc. Such specification may be made at different levels (national, sectoral, regional and local) for calculation of low carbon targets.

\section{Step 3: Identification of low carbon development paths taking account of availability of capitals and technologies}

The calculation of quantitative targets will include the following components: (1) Voluntary reductions whereby the country or industrial sector will plan or assume energy efficiency improvement for the planned period, given the resources and technologies at its disposal; (2) Conditional reductions which refers to the extra carbon savings that can be made if technology and financial assistance is made available through external sources; and (3) Obligatory reductions which means obligation made to avoid or restrict all wasteful and luxurious consumption/emissions. These would require the rejection of some development projects/emissions.

\section{Comparing the human development approach with the Kyoto Protocol}

There are a number of similarities and differences between Kyoto and the human development based approach to defining commitments suggested here. The principle is the same, i.e. common but differentiated responsibilities. Both approaches are also in line with ultimate objective defined in Article 2 of the Convention, aimed at stabilising atmospheric concentrations without harming the environment and development. However, some of the differences between the two approaches are fundamental.

1. The basis of Kyoto commitment is direct restriction of GHG emissions through allocating quotas to individual Annex I Parties. Under the human development approach, however, the basis is human development goals that are further translated into emission implications and commitments made without lowering human development goals. Therefore, the human development approach creates win-win solutions rather than the zero sum results under Kyoto style targets.

2. Methodology: the Kyoto approach is basically a top-down framework. By contrast, the country- 
driven and country-specific human development approach is more practical as the reductions of emissions do not follow any top-down requirement but rely on the potentials at project, sector and then economy-wide levels.

3. Separation of basic needs and luxurious/wasteful emissions: Kyoto commitment does not explicitly discourage luxurious/wasteful emissions. The human development approach by contrast attempts to guarantee emissions for basic human needs while discouraging luxurious/wasteful emissions.

4. Incentive/financial mechanisms: under Kyoto, incentives come from buying and selling "Kyoto units" under emissions trading, joint implementation and the clean development mechanism (CDM). The price of Kyoto units is intended to be uniform and there is no price discrimination against excessive consumptions. With the human development framework, a progressive taxation system is suggested, providing incentives for discouraging excessive emissions and a fair and effective financing mechanism for low carbon technologies.

5. Type of commitment: the Kyoto commitment is legally binding, but the human development based commitment offers the flexibility for both voluntary and conditional reductions. In addition, a third type of commitment is also proposed in the form of a moral obligation to restricting excessive emissions - an element, which is missing in Kyoto.

6. Environmental integrity: the realisation of Kyoto type targets relies on Parties to honour their commitments. Under human development, as low carbon development paths can generate considerable reductions, Parties would do their best to reach their goals to have a better image, domestically and internationally. The actual effects could be even better as no Party would then want to be seen as reneging from their commitments.

7. Participation: so far, under Kyoto only developed country Parties are required to commit to GHG reductions with developing countries being exempted from any quantitative limitations. The human development approach is primarily concerned with developing country participation and allows voluntary and conditional commitments by developed nations. Therefore, the low carbon development approach involves much wider participation than the Kyoto Protocol.

8. Hot air: under Kyoto, a base year has to be selected for proportional or relative reductions, thus creating the possibility of hot air if there is subsequently a non-climate policy related recession or economic downturn. In low carbon development, the goals are linked to human development. All carbon reductions are assessed against planned goals of human development. Therefore, no hot air would be created.

9. Flexibility/cost issues: under Kyoto, three flexibility mechanisms are initiated for cost-effective implementation of GHG reductions. But the carbon market involves complicated political processes, which could limit the scope for costeffective reductions. Under human development, by contrast, incentives are intrinsic for voluntary and conditional reductions. Industries and enterprises together with consumers can achieve energy efficiency goals without carbon prices.

\subsection{Incentives and disincentives for achieving emissions reductions}

Turning theoretical approaches into policies that achieve effective emissions reductions requires both carrots and sticks. In international negotiations, in most cases, sticks do not work well as a Party can choose to withdraw from the regime or renege on its commitments. Therefore, incentives play a more important and crucial role in the achievement of international commitments.

1. Emissions trading: in principle, voluntary reductions should be considered a baseline and the result of no-regret policies should therefore be ineligible for trading. Only reductions achieved that qualify as conditional should be tradeable. For the obligatory part, credits should be granted only for the reduction achieved from restricting luxurious emissions. These conditions for trading incentivise countries to make voluntary, conditional and obligatory commitments.

2. Conditionality requirement: reductions must not lower any development goals. This requirement is stipulated to guarantee that development goals take priority and to avoid over-estimate of emissions reductions.

3. Progressive taxation on emissions: to discourage excessive emissions, a progressive taxation on 
emissions is proposed here. If the emissions level is at basic needs level, a normal or basic rate is employed. Afterwards, as emissions increase, increasingly higher rates will be levied.

4. No exemption of luxurious emissions: no matter whether they take place in a rich or poor country, the assessment of development goals and the use of progressive taxation on emissions should be fully applicable to all countries - developed and developing.

\section{Conclusions}

The Kyoto Protocol has been ratified by many countries signifying a broad acceptance of its approach. However, the Kyoto approach also has limitations that have made it unattractive to some key players and, as argued in this article, the human development approach outlined here carries many advantages. Countries are encouraged under the human development approach to participate in emissions reductions on a no-risk basis as the basis of emissions reduction is linked to human development rather than carbon limits. In addition to this fundamental advantage, there are also a number of practical merits, such as allowing for a fuller consideration of national circumstances, basic needs satisfaction, international cooperation, and incentive mechanisms for environmentally sound trading.

\section{Note}

* The author would like to acknowledge with gratitude the kind assistance by Xianli Zhu in preparing this article and participants at various workshops on human development and emissions, Taishi Sugiyama, Henrik Hasselknippe, Feng Gao, Liyan Li, and Xuedu Lu, among many others, for their comments and suggestions. All the views and errors are the sole responsibility of the author.
The human development approach is also not a panacea and many conceptual and practical problems need further work. The first one being the difficulty in defining a clear and widely acceptable definition/specification of basic needs satisfaction. It is suggested that emissions for public goods and services, including roads, railways, airports, flood control and drainage systems, water supply and wastewater treatment facilities, for example, can be relatively easy to clarify. Emissions for basic needs relating to food production from the agricultural sector, housing, residential energy and transport are more difficult to calculate and agree.

A final weakness is that luxurious/wasteful emissions are not eliminated outright but become subject to political discussion. Because such emissions are taxed under this approach, additional political discussions and problems can be expected about the use of the funds raised through such a tax system. There are valid issues requiring further work but the important point here is that the concepts used in the human development approach can serve as a basis for further negotiations. To the extent they seem agreeable to a wide group of policy makers, more detailed work to elaborate models and figures can be undertaken - just as it was with Kyoto-style targets. 


\section{References}

Aldy, J., Ashton, J., Baron, R., Bodansky, B., Charnovitz, S., Diringer, E., Heller, T., Pershing, J., Shukla, P., Tubiana, L., Tudela, F. and Wang, X., 2003, Beyond Kyoto: Advancing the International Effort Against Climate Change, P. Pew Centre on Global Climate Change, November, www.pewclimate.org/global-warming-in-depth/ international/working_papers/beyondkyoto.cfm

Baumert, K.A. (ed.), 2002, Building on the Kyoto Protocol: Options for Protecting the Climate, World Resources Institute

Hoehne, N., Galleguillos, C., Blok, K., Harnisch, J. and Phylipsen, D., 2003, Evolution of Commitments under the UNFCCC: Involving Newly Industrialized Economies and Developing Countries, ECOFYS GmbH, on behalf of the German Federal Environmental Agency, February
Pan, J., 2003, 'Emissions rights and their transferability: equity concerns over climate change mitigation', International Environmental Agreements: Politics, Law and Economics, Vol 3 No 1: $1-16$

Pan, J., 2002, 'Rural energy patterns in China: a preliminary assessment of available data sources', paper presented at Stanford-TERI (The Energy Research Institute) 'Workshop on Rural Energy Transition and Sustainable Development', 5-8 November, New Delhi, Working Paper, Stanford Programme on Energy and Sustainable Development, pesd.stanford.edu/events/index.html Zhou, D., Dai, Y., Yi, C., Guo, Y. and Zhu, Y., 2003, China's Sustainable Energy Scenarios in 2020, Beijing: China Environmental Science Press 